\title{
Extratos aquosos de Psychotria sp. interferem na biologia de Plutella xylostella
}

\author{
Aqueous extracts of Psychotria sp. interfere with the biology of Plutella xylostella \\ Extractos acuosos de Psychotria sp. interfieren con la biología de Plutella xylostella
}

Recebido: 06/10/2021 | Revisado: 14/10/2021 | Aceito: 22/10/2021 | Publicado: 23/10/2021

\author{
Rosicleia Matias da Silva \\ ORCID: https://orcid.org/0000-0001-8988-1948 \\ Universidade Federal da Grande Dourados, Brasil \\ E-mail: rosi-matias09@hotmail.com \\ Claudemir Antonio Garcia Fioratti \\ ORCID: https://orcid.org/0000-0002-5719-1456 \\ Universidade Federal da Grande Dourados, Brasil \\ E-mail: claufioratti.ento@gmail.com \\ Eduardo Carvalho Faca \\ ORCID: https://orcid.org/0000-0002-2718-1871 \\ Universidade Federal da Grande Dourados, Brasil \\ eduardofaca@gmail.com \\ Rosilda Mara Mussury \\ ORCID: https://orcid.org/0000-0002-8961-9146 \\ Universidade Federal da Grande Dourados, Brasil \\ E-mail: mussuryufgd@gmail.com
}

\begin{abstract}
Resumo
Extratos botânicos com propriedades inseticidas buscam minimizar os problemas provocados pelo uso incorreto e muitas vezes exagerado dos agrotóxicos. Dependendo da concentração utilizada e do método de extração, certos extratos podem provocar diversas reações sobre os insetos-daninhos. Sendo assim, o presente trabalho teve por objetivo avaliar a toxicidade dos extratos aquosos elaborados por maceração e infusão de Psychotria capillacea, Psychotria deflexa e Psychotria leiocarpa sobre ovos, larvas e pupas de Plutella xylostella. Foram utilizados sete tratamentos para avaliar a toxicidade dos extratos, sendo: 1) infusão $P$. capillacea, 2) infusão de $P$. deflexa, 3 ) infusão de $P$. leiocarpa, 4) maceração P. capillacea, 5) maceração de $P$. deflexa, 6) maceração de $P$. leiocarpa e 7) água destilada (controle). Larvas de terceiro instar de P. xylostella e pupas com até três dias de formação receberam aplicação tópica dos tratamentos, enquanto fragmentos de couve contendo ovos desse inseto foram imersos nos tratamentos. Os resultados demonstraram que os extratos aquosos por infusão e maceração de $P$. capillacea e $P$. leiocarpa e $P$. deflexa não apresentaram toxicidade significativa sobre as larvas e pupas de $P$. xylostella, enquanto extratos elaborados por maceração reduziram a sobrevivência de ovos desse inseto. Observou-se que os extratos macerados causaram a mortalidade nas primeiras 46 horas e as infusões foram mais efetivas após esse período. Sendo assim, outros testes devem ser realizados para elucidar a eficiência dos extratos de Psychotria sobre os parâmetros biológicos e comportamentais de P. xylostella, tendo em vista que novas técnicas de extração devem ser exploradas.
\end{abstract}

Palavras-chave: Rubiaceae; Traça-das-crucíferas; Toxicidade; Larvicida; Pupicida; Ovicida.

\begin{abstract}
Botanical extracts with insecticidal properties seek to minimize the problems caused by the incorrect and often exaggerated use of pesticides. Depending on the concentration used and the extraction method, certain extracts can provoke different reactions on the harmful insects. Therefore, this study aimed to evaluate the toxicity of aqueous extracts prepared by maceration and infusion of Psychotria capillacea, Psychotria deflexa and Psychotria leiocarpa on eggs, larvae and pupae of Plutella xylostella. Seven treatments were used to evaluate the toxicity of the extracts: 1) $P$. capillacea infusion, 2) $P$. deflexa infusion, 3) $P$. leiocarpa infusion, 4) $P$. capillacea maceration, 5) $P$. deflexa maceration, 6) of $P$. leiocarpa maceration and 7) distilled water (control). Third-instar larvae of $P$. xylostella and pupae with up to three days of formation received topical application of the treatments, while fragments of cabbage containing eggs of this insect were immersed in the treatments. The results showed that the aqueous extracts by infusion and maceration of $P$. capillacea and P. leiocarpa and P. deflexa did not present significant toxicity on P. xylostella larvae and pupae, while the extracts prepared by maceration reduced the survival of eggs of this harmful insect. It was observed that macerated extracts caused mortality in the first 46 hours and infusions were more effective after this period. Therefore, other tests must be carried out to elucidate the efficiency of Psychotria extracts on the biological and behavioral parameters of $P$. xylostella, considering that new extraction techniques must be explored.
\end{abstract}

Keywords: Rubiaceae; Diamondback moth; Toxicity; Larvicide; Pupicide; Ovicide. 


\begin{abstract}
Resumen
Los extractos botánicos con propiedades insecticidas buscan minimizar los problemas causados por el uso incorrecto y muchas veces exagerado de plaguicidas. Dependiendo de la concentración utilizada y el método de extracción, ciertos extractos pueden provocar diferentes reacciones en los insectos dañinos. Por tanto, este estudio tuvo como objetivo evaluar la toxicidad de extractos acuosos preparados por maceración e infusión de Psychotria capillacea, Psychotria deflexa y Psychotria leiocarpa en huevos, larvas y pupas de Plutella xylostella. Se utilizaron siete tratamientos para evaluar la toxicidad de los extractos: 1) infusión de $P$. capillacea, 2) infusión de $P$. deflexa, 3) infusión de $P$. leiocarpa, 4) maceración de $P$. capillacea, 5) maceración de $P$. deflexa, 6) maceración de $P$. leiocarpa y 7) agua destilada (testigo). Las larvas de tercer estadio de $P$. xylostella y pupas con hasta tres días de formación recibieron aplicación tópica de los tratamientos, mientras que en los tratamientos se sumergieron fragmentos de col berza que contenían huevos de este insecto. Los resultados mostraron que los extractos acuosos por infusión y maceración de P. capillacea, P. leiocarpa y $P$. deflexa no presentaron toxicidad significativa sobre larvas y pupas de $P$. xylostella, mientras que los extractos preparados por maceración redujeron la supervivência de huevo de insectos. Se observó que los extractos macerados causaron mortalidad en las primeras 46 horas y las infusiones fueron más efectivas después de este período. Por tanto, se deben realizar otras pruebas para dilucidar la eficacia de los extractos de Psychotria sobre los parámetros biológicos y de comportamiento de $P$. xylostella, considerando que se deben explorar nuevas técnicas de extracción.
\end{abstract}

Palabras clave: Rubiaceae; Polilla crucífera; Toxicidad; Larvicida; Pupicida; Ovicida.

\title{
1. Introdução
}

A revolução verde trouxe grandes avanços para humanidade, como por exemplo a eficiência no campo e o aumento significativo na produção de alimentos (Brum, 1988; Sachs, 2009). Entretanto, o uso acentuado e incorreto dos agrotóxicos tornou-se um dos maiores problemas para a agricultura, principalmente no que diz respeito aos impactos negativos que essas substâncias tem causado sobre a biodiversidade (Carvalho, 2017). Outro efeito colateral da utilização errônea desses compostos químicos é a possibilidade de fazer com que os insetos-daninhos desenvolvam resistência aos seus princípios ativos, tornandoos ineficazes para o controle (Miller et al., 2010; Sparks \& Nauen, 2015).

Os extratos botânicos com propriedades inseticidas buscam minimizar esses problemas, pois não são letais para uma variedade de indivíduos não-alvo. Dependendo da concentração utilizada e do método de extração, certos extratos podem provocar diversas reações sobre os insetos-daninhos, como inibição da alimentação e da oviposição (Prakash \& Rao, 1997; Miresmailli \& Isman, 2014), redução da fecundidade (Peres et al. 2017; Ferreira et al. 2020), repelência (Boiça Jr. 2013), alterações morfológicas e fisiológicas (Ribeiro et al. 2016; Peres et al. 2017), toxicidade sobre larvas, pupas e ovos (Risco et al., 2012; Liu et al., 2019; Fernandes et al., 2021) e infertilidade em adultos (Santiago et al., 2008).

Alguns insetos são considerados polinizadores fundamentais para várias espécies de plantas, enquanto outros se tornaram inimigos por parasitarem e destruírem grandes plantações, como é o caso da Plutella xylostella L. 1758 (Plutellidae: Lepidoptera), popularmente conhecida por traça-das-crucíferas. Esse microlepidoptero é um dos principais inseto-daninho dos cultivares de brassicas, que pode gerar um prejuízo mundial de 4 a 5 bilhões de dólares anualmente com o manejo e produções de brássicas perdidas (Zalucki et al., 2012). Nos últimos dez anos, diversas plantas foram testadas quanto à atividade inseticida frente aos parâmetros biológicos de P. xylostella, entre elas Amaranthaceae, Acoraceae, Piperaceae, Solanaceae, Annonaceae, Fabaceae, Euphorbiaceae, Crassulaceae, Meliaceae, Asteraceae, Poaceae e Rubiaceae (Trindade et al., 2011; Couto et al., 2016; Hidayati et al., 2016; Boo et al., 2018; Fonseca et al., 2018; Matharu \& Mehta, 2018; Ahmad et al., 2019; Cerda et al., 2019; Karimzadeh \& Rabiei, 2020; Ningsih et al., 2020; Da Silva et al., 2020; Mpumi et al., 2020). Sendo essa última família de grande importância econômica e terapêutica no Brasil (Valli et al., 2016).

Rubiaceae é considerada a quarta maior família de Angiospermas do Brasil, com 1.415 espécies amplamente distribuída entre Amazônia, Caatinga, Cerrado, Mata Atlântica, Pampa e Pantanal (Barbosa et al., 2015; BFG, 2015). Algumas espécies de Rubiaceae apresentam alto grau de toxicidade, devido a presença de flavonoides, iridoides, alcaloides, terpenos, fenóis, saponinas, taninos, ésteres de ácido cafeico e compostos triterpenos oleanólico e ursólico (Silva et al., 2008; Matos et al., 2011; Valli et al., 2016; Peres et al., 2017). Estudos anteriores demonstraram que o extrato metanólico de Psychotria poeppigiana 
Mull. Arg. (Rubiaceae) promoveu a mortalidade de 45\% dos adultos de Sitophilus zeamais Mots. (Coleoptera: Curculionidae) no bioensaio por ingestão e 5\% de mortalidade no teste por contato (Silva et al., 2013). Enquanto os extratos etanólicos de Psychotria hoffmannseggiana (Roem. \& Schult.) Müll. Arg., Psychotria prunifolia (Kunth) Steyerm e Psychotria goyazensis Müll.Arg. apresentaram atividade ovicida sobre Spodoptera frugiperda (Lepidoptera: Noctuidae) (Souza Júnior et al., 2011).

Considerando o potencial inseticida demonstrado com os extratos de Psychotria sp. sobre insetos-daninhos, levantamos a hipótese de que algumas espécies desse gênero apresentam toxicidade sobre $P$. xylostella. Sendo assim, o presente trabalho teve por objetivo avaliar a toxicidade dos extratos aquosos de Psychotria capillacea (Müll. Arg.) Standl., Psychotria deflexa DC. e Psychotria leiocarpa Cham. \& Schltdl, elaborados por maceração e infusão, sobre ovos, larvas e pupas de $P$. xylostella.

\section{Metodologia}

A preparação dos extratos, manutenção da criação de $P$. xylostella e os experimentos foram realizados no Laboratório de Interação Inseto-Planta, localizado na Universidade Federal da Grande Dourados, Mato Grosso do Sul, Brasil, sob condições experimentais com controle de temperatura $\left(25 \pm 2{ }^{\circ} \mathrm{C}\right)$, umidade relativa $(60 \pm 5 \%)$ e fotoperíodo (12 horas).

\subsection{Material vegetal e preparação dos extratos}

A metodologia utilizada na obtenção dos extratos aquosos foi adaptada de Peres et al. (2017) e Barbosa et al. (2020). Folhas de $P$. leiocarpa, P. capillacea e $P$. deflexa, foram coletadas durante o período da manhã na Mata do Azulão, Fazenda Coqueiro no município de Dourados (22 $\left.12^{\prime} \mathrm{S} ; 54 \mathrm{o} 54^{\prime} \mathrm{W}\right)$. As espécies utilizadas foram identificadas pela Dra. Zefa Valdivina Pereira e uma exsicata de cada planta se encontra depositada no herbário da Universidade Federal da Grande Dourados (UFGD): Psychotria leiocarpa (DDMS 5007), Psychotria deflexa (DDMS 5005) e Psychotria capillacea (DDMS 5008).

Os extratos foram preparados por meio de duas técnicas distintas de extração: infusão e maceração. Para obtenção do macerado, em temperatura ambiente, misturou-se $10 \mathrm{~g}$ de folhas trituradas em $100 \mathrm{~mL}$ de água destilada. Essa solução foi agitada manualmente e refrigerada por 24 horas, a fim de se extrair os compostos hidrossolúveis. Posteriormente, foi filtrada com o auxílio de papel filtro para a obtenção do extrato aquoso na concentração (peso/volume) de 10\%. Para obtenção do extrato por infusão, misturou-se $10 \mathrm{~g}$ do material vegetal triturado em $100 \mathrm{~mL}$ de água destilada aquecida. Após agitação manual, a solução foi tampada e deixada em repouso por 15 minutos à temperatura ambiente $\left(25 \pm 2{ }^{\circ} \mathrm{C}\right)$. $\mathrm{O}$ extrato foi filtrado com o auxílio de papel filtro para a obtenção do extrato por infusão na concentração (peso/volume) de $10 \%$.

Assim, os tratamentos utilizados no ensaio foram: 1) infusão $P$. capillacea, 2) infusão de $P$. deflexa, 3) infusão de $P$. leiocarpa, 4) maceração $P$. capillacea, 5) maceração de $P$. deflexa, 6) maceração de $P$. leiocarpa e 7) água destilada (controle).

\subsection{Criação e manutenção de $P$. xylostella}

Indivíduos de P. xylostella foram coletados em hortas orgânicas no município de Dourados-MS. Posteriormente, foram transportados para o Laboratório de Interação Inseto-Planta (LIIP). As larvas foram acondicionadas em recipiente plástico (30 x $15 \times 12 \mathrm{~cm}$ ) e alimentadas com couve orgânica (Brassica oleracea var. acephala DC), previamente higienizada com solução de hipoclorito a 5\% e água corrente. A manutenção dos recipientes de criação foi feita diariamente até a formação das pupas, que foram retiradas e transferidas para gaiolas de plástico $(9$ x 19 x $19 \mathrm{~cm})$. Os adultos que emergiram foram alimentados com solução de mel a 10\% e para a obtenção das posturas, foi inserido no interior das gaiolas discos de couve orgânica e de papel filtro. Após a oviposição, os discos com ovos foram transferidos para o recipiente plástico contendo couve orgânica (Barros et al., 2012). 


\subsection{Toxicidade dos extratos sobre larvas e pupas de P. xylostella}

A metodologia para avaliação da toxicidade dos extratos aquosos em larvas e pupas de $P$. xylostella foi adaptada de Risco et al. (2012), Hernández-Lambraño et al. (2014), Huang et al. (2014) e Santos et al. (2015). Dez pupas com até três dias de formação e dez larvas de terceiro instar de P. xylostella receberam aplicação tópica dos tratamentos assinalados no item 2.1. Posteriormente, após secas em temperatura ambiente por 20 minutos, as pupas e larvas foram transferidas para uma placa de Petri $(12 \times 2 \mathrm{~cm})$ contendo discos de couve orgânica e monitoradas a cada 24 horas por quatro dias (Figura 1A). Enquanto as pupas foram transferidas para uma gaiola de plástico $(100 \times 10 \times 12 \mathrm{~cm})$ e monitoradas por 7 dias, para registrar a sobrevivência de larvas e pupas, por meio da contagem das larvas que permaneceram vivas e pelo número de adultos emergidos, respectivamente (Figura 1B). As larvas foram consideradas mortas se não exibissem nenhuma resposta observável a um estímulo mecânico, ou seja, pressão de curto prazo aplicada com um pincel.

Figura 1. Esquema representativo do teste de toxicidade dos extratos sobre larvas (A) e pupas (B) de P. xylostella. Experimento desenvolvido em condições controladas de temperatura $\left(25 \pm 2{ }^{\circ} \mathrm{C}\right)$, umidade relativa $(60 \pm 5 \%)$ e fotoperíodo (12 horas).

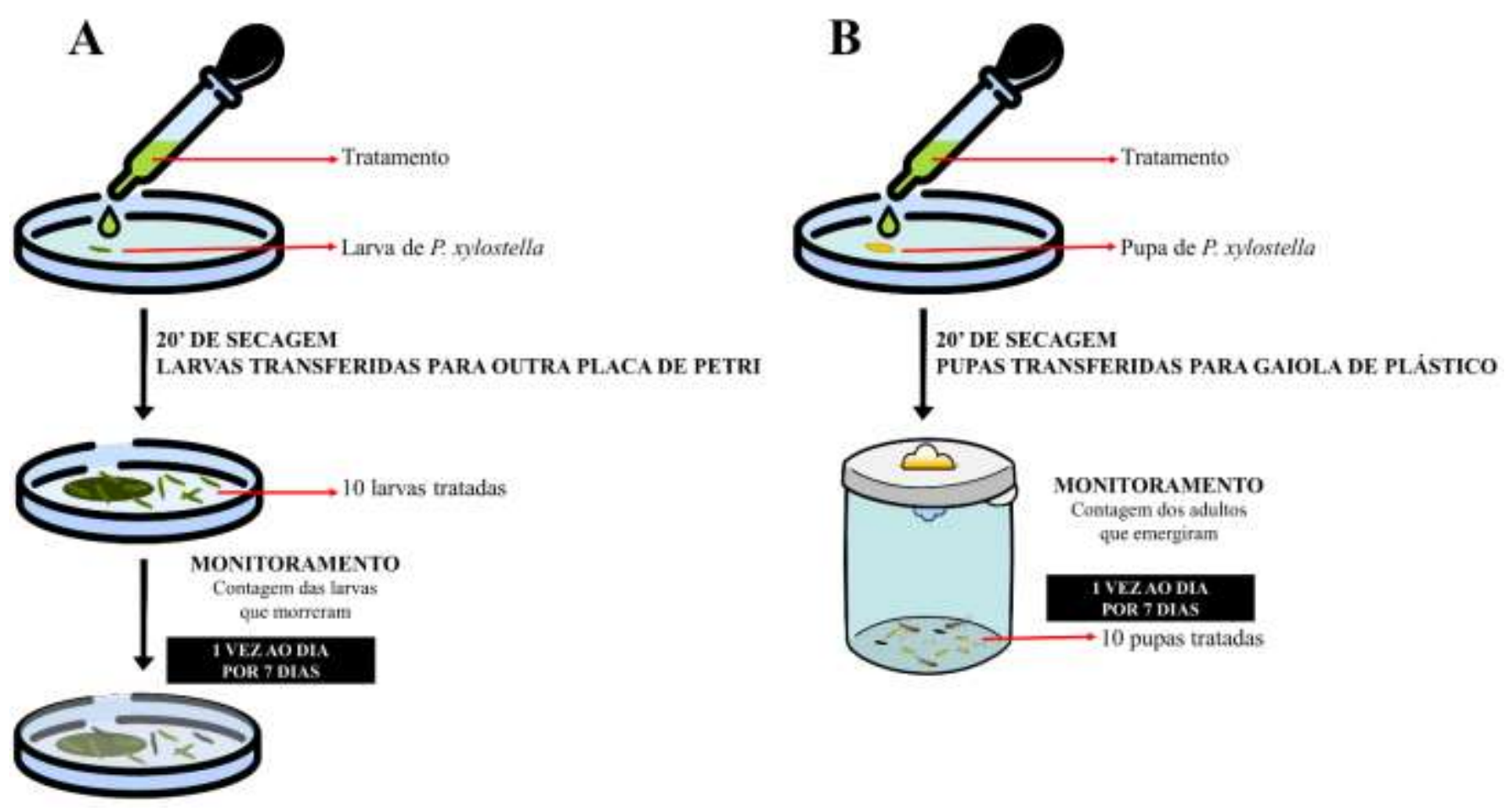

Fonte: Autores.

Cada tratamento foi composto por 10 repetições, tendo cada um 10 larvas ou 10 pupas de $P$. xylostella, totalizando 100 indivíduos por tratamento, como demonstrado na ilustração acima.

\subsection{Toxicidade dos extratos sobre ovos de $P$. xylostella}

A metodologia utilizada neste experimento foi baseada em Liu et al. (2019). Cinco casais de P.xylostella com até $12 \mathrm{~h}$ de emergência foram colocados em uma gaiola plástica $(10 \times 10 \times 12 \mathrm{~cm})$ contendo discos de couve e de papel filtro levemente umedecido com água destilada ( $8 \mathrm{~cm}$ de diâmetro). Após 24 horas de exposição, os discos foram retirados das gaiolas e cuidadosamente recortados, de modo que cada fragmento da folha de couve contivesse 10 ovos do inseto-daninho. 
Os fragmentos de couve foram imersos nos respectivos extratos vegetais e no controle (água destilada) por 10 segundos, e secos em temperatura ambiente por 20 minutos e, em seguida, transferidos para uma placa de Petri forrada com papel filtro. As placas foram fechadas e monitoradas diariamente por 10 dias, para registrar a sobrevivência dos ovos, por meio da contagem de larvas eclodidas (Figura 2). Cada tratamento foi composto por 10 repetições, tendo cada um 10 ovos de P. xylostella, totalizando 100 ovos.

Figura 2. Esquema representativo do teste de toxicidade dos extratos sobre ovos de P. xylostella. Experimento desenvolvido em condições controladas de temperatura $\left(25 \pm 2{ }^{\circ} \mathrm{C}\right)$, umidade relativa $(60 \pm 5 \%)$ e fotoperíodo (12 horas).

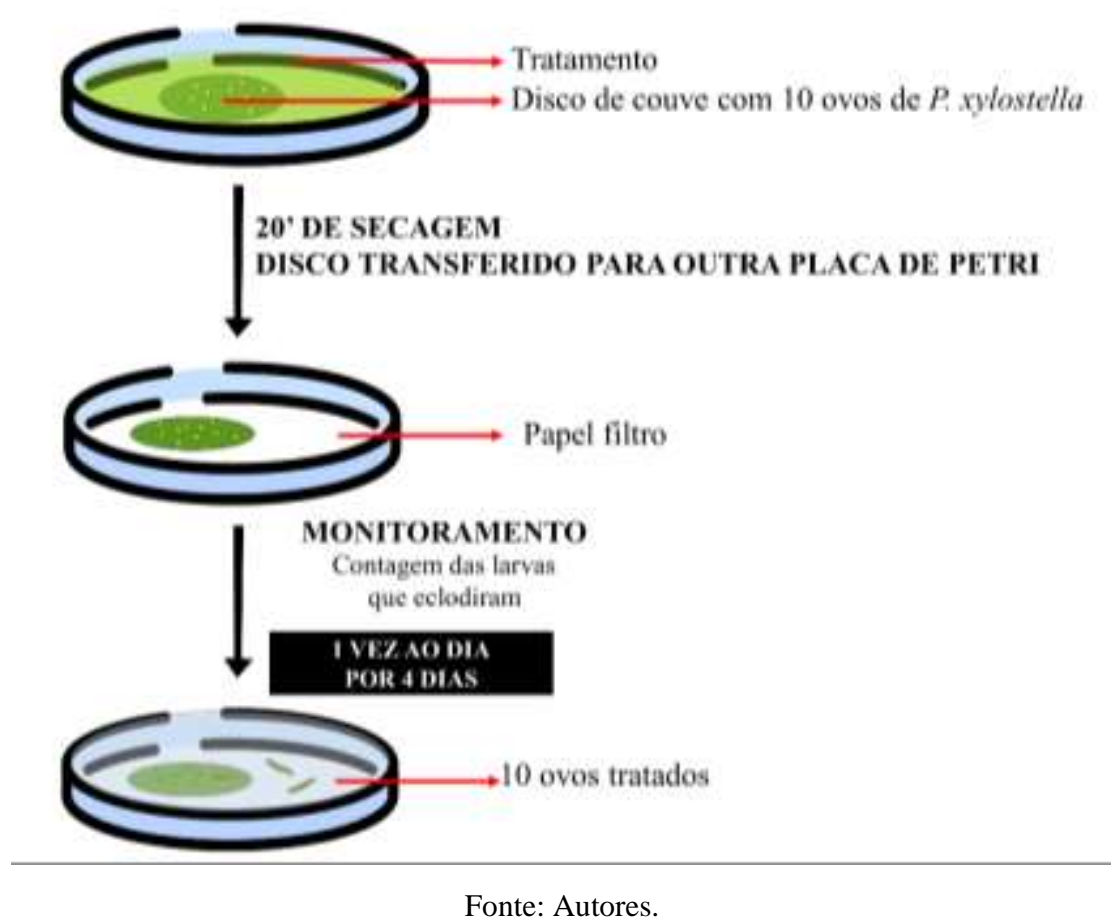

Cada tratamento foi composto por 10 repetições, tendo em cada um 10 ovos de P. xylostella, totalizando 100 ovos, como demonstrado na ilustração acima.

\subsection{Análise Estatística}

O experimento foi conduzido em delineamento inteiramente casualizado em esquema fatorial 4 x 2 (planta x métodos de extração). O conjunto de dados foi submetido ao teste de normalidade e homogeneidade. Os valores que não atenderam aos pressupostos foram transformados para arcoseno da $\sqrt{ } \mathrm{x} / 100$. Após cumpridos os pressupostos, os dados foram submetidos à análise de variância (ANOVA) e sendo constatado significância entre os tratamentos, aplicou-se teste de comparação de médias (Tukey a 5\% de probabilidade) com auxílio do software R (R Core Team 2020).

\section{Resultados e Discussão}

\subsection{Toxicidade sobre as larvas e pupas de $P$. xylostella}

Não houve significância nos fatores isolados e nem interação entre os fatores na variável sobrevivência das larvas. Foi observado que os extratos aquosos de $P$. capillacea e $P$. leiocarpa e $P$. deflexa elaborados por infusão e maceração não apresentaram toxicidade significativa sobre as larvas de P. xylostella (Tabela 1). Tal achado pode estar relacionado ao método de aplicação da substância, ou seja, alguns compostos químicos são mais eficazes quando aplicados diretamente no inseto, 
enquanto outros compostos produzem toxicidade por meio da ingestão dessas substâncias (Gosselin, 1984). Além disso, a barreira natural presente na estrutura corporal do inseto pode ajudar a prevenir a absorção de produtos tóxicos e reduzir o efeito inseticida da aplicação tópica (Alecio, 2012).

Mesmo não demonstrando toxicidade tópica sobre as larvas de P. xylostella, trabalhos anteriores comprovaram que algumas espécies de Psychotria possuem toxicidade larval sobre outras espécies de lepidópteras. De acordo com Tavares et al. (2013), no teste por ingestão, os extratos etanólicos das folhas e caules de Psychotria hoffmannseggiana, Psychotria prunifolia., Psychotria goyazensis e Psychotria capitata Ruiz \& Pavon, apresentaram eficiência superior a $80 \%$ na mortalidade de larvas de Spodoptera frugiperda. Enquanto a ingestão do extrato etanólico das folhas de $P$. prunifolia provocou a mortalidade das larvas de Sitotroga cerealella Oliver 1819 (Lepidoptera: Gelechiidae) (Fouad et al., 2014). Os extratos etanólicos das folhas de P. leiocarpa, Psychotria brachyceras Müll. Arg., Psychotria umbellata Ruiz \& Pav. e Psychotria carthagenensis Jacq. causaram a mortalidade em larvas de Helicoverpa armigera (Hübner, 1805) (Lepidoptera: Noctuidae) quando se alimentaram com os extratos dessas plantas (Matsuura et al. 2016).

Tabela 1: Sobrevivência das larvas, pupas e ovos Plutella xylostella (média \pm EP) no fator isolado: Planta. Temperatura $25 \pm 2^{\circ} \mathrm{C}$, Umidade Relativa $60 \pm 5 \%$ e Fotoperíodo 12 horas. Dourados, MS. 2021.

\begin{tabular}{lccc}
\hline Espécie & Larvas & Pupas & Ovos \\
\hline Controle & $97 \pm 1.47 \mathrm{a}$ & $87 \pm 1.47 \mathrm{a}$ & $89 \pm 1.91 \mathrm{a}$ \\
& $n=200$ & $n=200$ & $n=200$ \\
P. capillacea & $91.5 \pm 1.96 \mathrm{a}$ & $85.5 \pm 2.11 \mathrm{a}$ & $61 \pm 6.53 \mathrm{~b}$ \\
& $n=200$ & $n=200$ & $n=200$ \\
$P$. deflexa & $94.5 \pm 1.53 \mathrm{a}$ & $85.5 \pm 1.53 \mathrm{a}$ & $58 \pm 6.08 \mathrm{~b}$ \\
& $n=200$ & $n=200$ & $60 \pm 5.28 \mathrm{~b}$ \\
$P$. leiocarpa & $92.5 \pm 1.60 \mathrm{a}$ & $84 \pm 2.34 \mathrm{a}$ & $n=200$ \\
& $n=200$ & $n=200$ & $\mathrm{~F}=16.6$ \\
\hline Valor de $\mathrm{F}$ & $\mathrm{F}=2.50$ & $\mathrm{~F}=0.17$ & $\mathrm{p} \leq 0.0001$ \\
& $\mathrm{p}=0.07$ & $\mathrm{p}=0.92$ & $\mathrm{GL}=3$ \\
& $\mathrm{GL}=3$ & $\mathrm{GL}=3$ & 34.0 \\
\hline
\end{tabular}

As médias seguidas por letras diferentes na mesma coluna diferem em nível de significância de 5\%.n= número de indivíduos avaliados.

Fonte: Autores.

Na tabela acima é possível observar que as variáveis sobrevivência das larvas e das pupas não apresentaram significância no fator isolado planta, sendo observado significância apenas na variável sobrevivência dos ovos. Os resultados também demonstraram que os extratos macerados causaram a mortalidade nas primeiras 46 horas, enquanto as infusões foram mais efetivas após esse período (Figura 3). 
Figura 3. Taxa de mortalidade das larvas de P. xylostella após a exposição aos extratos de A) Psychotria capillacea, B) Psychotria deflexa e C) Psychotria leiocarpa elaborados por maceração $(\boldsymbol{\Delta})$ e infusão (๑).
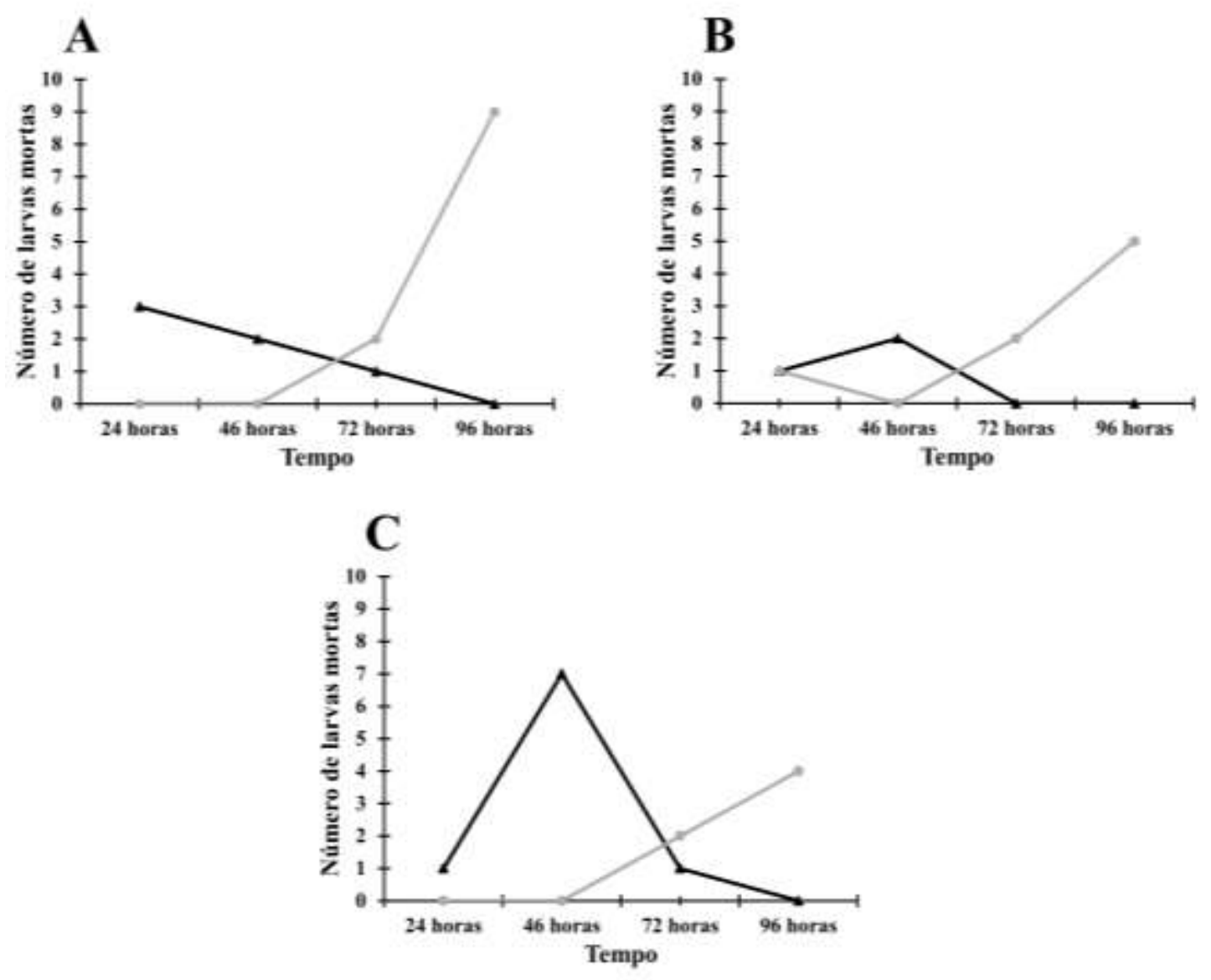

Fonte: Autores.

Observa-se na figura acima, que taxa mortalidade das larvas alimentadas com extratos macerados possuem uma tendencia decrescente, ou seja, com a passar das horas os extratos macerados reduziram ou estacionaram a mortalidade das larvas, enquanto as infusões apresentaram resultado inverso, onde os extratos aumentaram a mortalidade larval com o passar do tempo de observação. Tal observação pode ser explicada considerando que os inseticidas botânicos possuem composições químicas e modos de ação diferentes, ou seja, alguns compostos podem agir imediatamente sobre os insetos enquanto, outros podem levar mais tempo para agir (Borden et al., 2018).

No teste de toxicidade pupal, não houve significância no fator isolado planta e nem interação entre os fatores. Porém, foi observado significância no método de extração, onde as pupas de $P$. xylostella que receberam aplicação tópica dos extratos macerados apresentaram redução no número de adultos emergidos (Tabela 2). Existem poucos trabalhos que elucidam a toxicidade tópica de extratos de Rubiaceae, principalmente no que diz respeito a pupas de lepidópteros, entretanto, o extrato etanólico do caule de Coutarea hexandra (Jacq.) K. Schum. apresentou toxicidade tópica sobre pupas de Aedes Aegypti L., 1762 (Diptera: Culicidae) (Candido et al. 2013), em contrapartida, o extrato etanólico das folhas de Genipa americana L. não demonstrou atividade pupicida sobre esse inseto (Barbosa et al., 2014).

Em trabalhos anteriores, foi avaliada a sobrevivência pupal de indivíduos alimentados com extratos de Rubiaceae na fase larval. O extrato aquoso de Alibertia sessilis (Vell.) K. Schum. reduziu a sobrevivência pupal de P. xylostella em 60\%, enquanto os extratos aquosos de A. edulis e A. intermedia e os extratos etanólicos de A. edulis, A. sessilis e A. intermedia não 
demonstraram influência sobre a sobrevivência dessas pupas (Peres et al., 2017; Da Silva et al., 2020). Os extratos metanólicos das folhas de Palicourea rigida Kunth. e Rudgea viburnoides (Cham.) não influenciaram na sobrevivência pupal de S. frugiperda (Alves et al., 2018). Esses resultados contrastantes comprovam que a atividade tóxica dos extratos depende da espécie de inseto estudado, solvente utilizado na extração dos compostos e no método de aplicação dessas substâncias.

Tabela 2: Sobrevivência das larvas, pupas e ovos Plutella xylostella (média \pm EP) no fator isolado: Método de Extração. Temperatura $25 \pm 2^{\circ} \mathrm{C}$, Umidade Relativa $60 \pm 5 \%$ e Fotoperíodo 12 horas. Dourados, MS. 2021.

\begin{tabular}{|c|c|c|c|}
\hline Método de Extração & Larvas & Pupas & Ovos \\
\hline \multirow[t]{2}{*}{ Infusão } & $93 \pm 1.25 \mathrm{a}$ & $87.2 \pm 2.13 \mathrm{a}$ & $84 \pm 2.08 \mathrm{a}$ \\
\hline & $n=400$ & $n=400$ & $n=400$ \\
\hline \multirow[t]{2}{*}{ Maceração } & $94.8 \pm 1.13 \mathrm{a}$ & $83.8 \pm 2.13 b$ & $50.3 \pm 4.04 \mathrm{~b}$ \\
\hline & $n=400$ & $n=400$ & $n=400$ \\
\hline \multirow[t]{3}{*}{ Valor de F } & $\mathrm{F}=1.07$ & $\mathrm{~F}=5.37$ & $\mathrm{~F}=82.2$ \\
\hline & $\mathrm{p}=0.31$ & $\mathrm{p}=0.02$ & $\mathrm{p} \leq 0.0001$ \\
\hline & $\mathrm{GL}=1$ & $\mathrm{GL}=1$ & $\mathrm{GL}=1$ \\
\hline $\mathrm{CV}(\%)$ & 13.7 & 13.2 & 34.0 \\
\hline
\end{tabular}

As médias seguidas por letras diferentes na mesma coluna diferem em nível de significância de 5\%. n= número de indivíduos avaliados.

Fonte: Autores.

Na tabela acima, observa-se que o método de extração dos extratos apresenta significância nas variáveis sobrevivência das pupas e dos ovos, enquanto a sobrevivência das larvas não foi influencias pelos método de extração.

\subsection{Toxicidade sobre ovos de $P$. xylostella}

Foi observado interação significativa entre os fatores para a variável sobrevivência de ovos, onde todos os extratos macerados das espécies testadas apresentaram toxicidade significativa sobre os ovos de $P$. xylostella, enquanto as infusões não diferiram do controle (Tabela 3). De acordo com Fonsêca (2005), a eficiência dos extratos também pode ser influenciada pelo tempo de preparo e a temperatura utilizada durante a extração. A maceração realizada nesse experimento foi de 24 horas com água destilada em temperatura ambiente, enquanto na infusão o tempo de extração foi de 15 minutos e com água fervente, logo, a maceração teve mais tempo para extrair os compostos bioativos das plantas e a alta temperatura do solvente, na infusão, pode ter provocado a degradação de alguns desses compostos químicos (Shaidi \& Naczk, 1995).

Vale ressaltar que o tipo de solvente empregado e o órgão vegetal utilizado também influenciam na eficácia dos extratos (Fonsêca, 2005). Em estudos anteriores, foi demonstrado que o extrato etanólico das folhas e caules de P. hoffmannseggiana, P. prunifolia, $P$. goyazensis e $P$. capitata causaram uma redução superior a $80 \%$ na eclosão das larvas de S. frugiperda (Souza Junior et al., 2011; Tavares et al., 2013), onde o extrato do caule de P. goyazensis reduziu em 100\% a taxa de eclosão dos ovos recém-depositados (Tavares et al., 2013).

A toxicidade de Rubiaceae também foi confirmada em ovos de outros indivíduos, entre eles, citamos o extrato aquoso e etanólico de Nauclea latifolia Sm. que reduziram a taxa de eclosão das larvas de Heligmosomoides bakeri (Nematoda: Heligmosomatidae) (Josué et al., 2012) e o extrato aquoso de Palicourea marcgravii A. St.-Hil. Que causou a mortalidade dos ovos da mosca-negra-dos-citros (Aleurocanthus woglumi Ashby (Hemiptera: Aleyrodidae)) (Pena, 2012). 
Tabela 3: Sobrevivência das larvas, pupas e ovos Plutella xylostella (média \pm EP) no desdobramento da interação planta x método de extração. Temperatura $24 \pm 1^{\circ} \mathrm{C}$, Umidade Relativa 73,3 $\pm 7 \%$ e Fotoperíodo 12 horas. Dourados, MS. 2021.

\begin{tabular}{|c|c|c|c|c|c|c|}
\hline \multirow{2}{*}{ Tratamento } & \multicolumn{2}{|c|}{ Larva } & \multicolumn{2}{|c|}{ Pupas } & \multicolumn{2}{|c|}{ Ovos } \\
\hline & Infusão & Maceração & Infusão & Maceração & Infusão & Maceração \\
\hline \multirow[t]{2}{*}{ Controle } & \multicolumn{2}{|c|}{$97 \pm 2.13 \mathrm{aA}$} & \multicolumn{2}{|c|}{$87 \pm 2.13 \mathrm{aA}$} & \multicolumn{2}{|c|}{$89 \pm 2.79 \mathrm{aA}$} \\
\hline & \multicolumn{2}{|c|}{$n=100$} & \multicolumn{2}{|c|}{$n=100$} & \multicolumn{2}{|c|}{$n=100$} \\
\hline P. capillacea & $\begin{array}{c}89 \pm 2.77 \mathrm{aA} \\
n=100\end{array}$ & $\begin{array}{c}94 \pm 2.67 \mathrm{aA} \\
n=100\end{array}$ & $\begin{array}{c}83 \pm 2.13 \mathrm{aA} \\
n=100\end{array}$ & $\begin{array}{c}88 \pm 3.59 \mathrm{aA} \\
n=100\end{array}$ & $\begin{array}{c}87 \pm 3.67 \mathrm{aA} \\
n=100\end{array}$ & $\begin{array}{c}35 \pm 4.01 \mathrm{bB} \\
n=100\end{array}$ \\
\hline P. deflexa & $\begin{array}{c}92 \pm 2.50 \mathrm{aA} \\
n=100\end{array}$ & $\begin{array}{c}97 \pm 1.53 \mathrm{aA} \\
n=100\end{array}$ & $\begin{array}{c}84 \pm 2.21 \mathrm{aA} \\
n=100\end{array}$ & $\begin{array}{c}87 \pm 2.13 \text { aA } n= \\
100\end{array}$ & $\begin{array}{c}81 \pm 4.33 \mathrm{aA} \\
n=100\end{array}$ & $\begin{array}{c}36 \pm 4.99 \mathrm{bB} \\
n=100\end{array}$ \\
\hline P. leiocarpa & $\begin{array}{c}94 \pm 2.21 \mathrm{aA} \\
n=100\end{array}$ & $\begin{array}{c}91 \pm 2.33 \mathrm{aA} \\
n=100\end{array}$ & $\begin{array}{c}81 \pm 2.77 \mathrm{aA} \\
n=100\end{array}$ & $\begin{array}{c}87 \pm 3.67 \mathrm{aA} \\
n=100\end{array}$ & $\begin{array}{c}79 \pm 5.26 \mathrm{aA} \\
n=100\end{array}$ & $\begin{array}{c}41 \pm 3.14 \mathrm{bB} \\
n=100\end{array}$ \\
\hline \multirow[t]{3}{*}{ Valor de F } & \multicolumn{2}{|c|}{$\mathrm{F}=1.46$} & \multicolumn{2}{|c|}{$\mathrm{F}=0.85$} & \multicolumn{2}{|c|}{$F=9.83$} \\
\hline & \multicolumn{2}{|c|}{$\mathrm{p}=0.23$} & \multicolumn{2}{|c|}{$\mathrm{p}=0.47$} & \multicolumn{2}{|c|}{$\mathrm{p} \leq 0.0001$} \\
\hline & \multicolumn{2}{|c|}{$\mathrm{GL}=3$} & \multicolumn{2}{|c|}{$\mathrm{GL}=3$} & \multicolumn{2}{|c|}{$\mathrm{GL}=3$} \\
\hline $\mathrm{CV}(\%)$ & \multicolumn{2}{|c|}{13.7} & \multicolumn{2}{|c|}{13.24} & \multicolumn{2}{|c|}{34.0} \\
\hline
\end{tabular}

As médias seguidas de letras distintas, maiúscula na coluna e minúscula na linha, diferem entre si a 5\% de probabilidade pelo teste de Tukey; $n=$ número de indivíduos analisados.

Fonte: Autores.

Na tabela acima é possível observar que houve interação entre os fatores para sobrevivência dos ovos. Esse resultado pode ser muito significativo no campo, pois a redução na taxa de eclosão larval interfere diretamente na próxima etapa do ciclo biológico, ou seja, no número de indivíduos na fase larval. Quanto menos larvas eclodem, menos alimento é consumido, reduzindo assim os danos nos cultivares de brássicas (Maroneze \& Gallegos, 2009).

\section{Conclusão}

Os extratos aquosos a $10 \%$ de $P$. leiocarpa, $P$. capillacea e $P$. deflexa elaborado por maceração possuem toxicidade sobre ovos de P. xylostella. As infusões não influenciaram na sobrevivência pupal, larval e dos ovos de $P$. xylostella. Sendo assim, encorajamos a continuidade dos estudos que buscam elucidar a eficiência dos extratos de Psychotria sobre os parâmetros biológicos (ciclo de vida) e comportamentais (oviposição e alimentação) de P. xylostella, tendo em vista que novas técnicas de extração devem ser exploradas.

Outro aspecto a ser avaliado é os efeitos no meio ambiente, dessa forma, novos estudos com concentrações diferentes e aplicação sobre organismos não alvo como inimigos natural, polinizadores, solo, água e ar devem ser estudados, bem como, aspectos relativos à toxidez dos extratos, para garantir a saúde do produtor.

\section{Agradecimentos}

Os autores agradecem ao Laboratório de Interação Inseto-Planta da Universidade Federal da Grande Dourados pelo apoio logístico, ao Conselho Nacional de Aperfeiçoamento da Educação Superior-Brasil (CAPES) pela bolsa de estudo da primeira autora e à Fundação de Apoio ao Desenvolvimento do Ensino, Ciência e Tecnologia do Estado de Mato Grosso do Sul (FUNDECT) pelo recurso disponibilizado pelo processo $\mathrm{n}^{\circ} 71 / 711.130 / 2018$. 


\section{Referências}

Ahmad, B., Mehmood, N., Sohail, K., Saljoqi, A. U. R., Khan, A., Rab, A., Zada, H., \& Hussain, S. (2019). In vitro management of Diamondback Moth (Plutella xylostella L.) using different concentrations of parthenium and neem extracts. J. Agr. Sci. Tech., 21(3), 659-669.

Alecio, M.R. (2012). Atividade biológica de extratos de Timbó (Derris scandens Aubl. e Deguelia floribundus Benth) sobre Cerotoma tingomarianus Bechyné (Coleoptera: Chrysomelidae) E Spodoptera frugiperda (J. E. Smith, 1797) (Lepidoptera: Noctuidae). Tese de Doutorado, Universidade Federal do Amazonas, Manaus, Amazonas, Brasil.

Alves, D. S., Carvalho, G. A., Oliveira, D. F., \& Corrêa, A. D. (2018). Screening of Brazilian plant extracts as candidates for the control of Spodoptera frugiperda (Lepidoptera: Noctuidae). Revista Colombiana de Entomologia, 44(1), 32-38. https://doi.org/10.25100/socolen.v44i1.6539

Barbosa, D. R. R., Boyarski, D. R. S., Macena, T. F. S., \& Clemente, R. C. (2020). Quantificação de compostos fenólicos, poder antioxidante e teor de açucares em produtos comerciais à base de Hibiscus sabdariffa L. Revista Desafios, 7(2), 108-123. https://doi.org/10.20873/uftv7-7770

Barbosa, M. R., Zappi, D., Taylor, C., Cabral, E., Jardim, J. G., Pereira, M. S., Calió, M. F., Pessoa, M. C. R., Salas, R., Souza, E. B., Di Maio, F. R., Macias, L., Anunciação, E. A., Germano Filho, P., Oliveira, J. A., Bruniera, C. P., Gomes, M., De Toni, K., \& Firens, M. (2015). Rubiaceae in lista de espécies da flora do Brasil. Jardim Botânico do Rio de Janeiro. http://floradobrasil.jbrj.gov.br/jabot/floradobrasil/FB210.

Barbosa, P. B. B. M., de Oliveira, J. M., Chagas, J. M., Rabelo, L. M. A., de Medeiros, G. F., Giodani, R. B., da Silva, E. A., Uchôa, A. F., \& Ximenes, M. F. F. M. (2014). Evaluation of seed extracts from plants found in the Caatinga biome for the control of Aedes aegypti. Parasitology Research, 113(10), 3565-3580. https://doi.org/10.1007/s00436-014-4022-6

Barros, R., Thuler, R. T., \& Pereira, F. F. (2012). Técnica de criação de Plutella xylostella (L. 1758) (Lepidoptera: Yponomeutidae). Em D. Pratissoli (Org.), Técnicas de Criação de Pragas de Importância Agrícola, em Dietas Naturais (pp. 65-84). Vitória, ES: Edufes.

BFG - The Brazil Flora Group et al. (2015). Growing knowledge: An overview of Seed Plant diversity in Brazil. Rodriguesia, 66(4), 1085-1113. https://doi.org/10.1590/2175-7860201566411

Boiça Júnior, A. L., Janini, J. C., de Souza, B. H. S., \& Rodrigues, N. E. L. (2013). Efeito de cultivares de repolho e doses de extrato aquoso de nim na alimentação e biologia de Plutella xylostella (Linnaeus) (Lepidoptera: Plutellidae). Bioscience Journal, 29(1), 22-31.

Boo, K. H., Seo, J. K., Ahn, J., Riu, K. Z., \& Kim, S. -I. (2018). The effect of Achyranthes japonica extract on larval survival and development and oviposition behavior of Plutella xylostella L. (Lepidoptera: Plutellidae). Journal of Asia-Pacific Entomology, 21(1), 29-36. https://doi.org/10.1016/j.aspen.2017.11.002

Borden, M. A., Buss, E. A., Park Brown, S. G., \& Dale, A. G. (2018). Natural products for managing landscape and garden pests in Florida. Edis, 2018(5), 113. https://doi.org/10.32473/edis-in197-2018

Brum, A. J. (1988). Modernização da agricultura: trigo e soja. Petrópolis: Vozes.

Candido, L. P., Cavalcanti, M. T., \& Beserra, E. B. (2013). Bioactivity of plant extracts on the larval and pupal stages of Aedes aegypti (Diptera, Culicidea). Revista da Sociedade Brasileira de Medicina Tropical, 46(4), 420-425. https://doi.org/10.1590/0037-8682-0118-2013

Carvalho, F. P. (2017). Pesticides, environment, and food safety. Food and Energy Security, 6(2), 48-60. https://doi.org/10.1002/fes3.108

Cerda, H., Carpio, C., Ledezma-carrizalez, A. C., Sánchez, J., Ramos, L., Muñoz-shugulí, C., Andino, M., \& Chiurato, M. (2019). Effects of aqueous extracts from amazon plants on Plutella xylostella (Lepidoptera: Plutellidae) and Brevicoryne brassicae (Homoptera: Aphididae) in laboratory, semifield and field trials. Journal of Insect Science, 19(5), 1-9. https://doi.org/10.1093/jisesa/iez068

Couto, I. F. S., Fuchs, M. L., Mauad, M., Scalon, S. P. Q., Dresch, D. M., \& Mussury, R. M. (2016). Feeding preference of Plutella xylostella for leaves treated with plant extracts. Anais da Academia Brasileira de Ciências, 88(3), 1781-1789.

Da Silva, R. M., Santos, L. P., Brito Silva, G., Miranda, L. O., Fioratti, C. A. G., Scalon, S. de P. Q., Mauad, M., \& Mussury, R. M. (2020). Alibertia spp. (Rubiaceae) extracts interfere with the development and reproduction of Plutella xylostella L. (Lepidoptera: Plutellidae). Gesunde Pflanzen, 72(4), 351-360. https://doi.org/10.1007/s10343-020-00517-3

Fouad, H. A., Faroni, L. R. D. A., Tavares, W. de S., Ribeiro, R. C., Freitas, S. de S., \& Zanuncio, J. C. (2014). Botanical extracts of plants from the Brazilian Cerrado for the integrated management of Sitotroga cerealella (Lepidoptera: Gelechiidae) in stored grain. Journal of Stored Products Research, 57, 6-11. https://doi.org/10.1016/j.jspr.2014.01.001

Fernandes, D. A., Rique, H. L., de Oliveira, L. H. G., Santos, W. G. S., de Souza, M. de F. V., \& da Cruz Nunes, F. (2021). Ovicidal, pupicidal, adulticidal, and repellent activity of Helicteres velutina K. Schum against Aedes aegypti L. (Diptera: Culicidae). Revista Brasileira de Medicina Veterinaria, 43, 1-15. https://doi.org/10.29374/2527-2179.BJVM112120

Ferreira, E. A., de Souza, S. A., Domingues, A., Da Silva, M. M. M., Padial, I. M. P. M., Carvalho, E. M. de, Cardoso, C. A. L., da Silva, S. V., \& Mussury, R. M. (2020). Phytochemical screening and bioactivity of Ludwigia spp. In the control of Plutella xylostella (Lepidoptera: Plutellidae). Insects, 11(9), 1-14. https://doi.org/10.3390/insects11090596

Fonseca, J., Couto, I. F. S., Da Silva Matias, R., Fioratti, C. A. G., Pereira, F. F., Mauad, M., De Paula Quintão Scalon, S., Carvalho, E. M., \& Mussury, R. M. (2018). Efeito de extratos metanólicos de Stryphnodendron adstringens (Mart) Coville na alimentação e reprodução de Plutella xylostella L. (Lepidoptera: Plutellidae). Interciencia, 43(3), 182-187.

Fonsêca, S. G. C. (2005). Farmacotécnica de fitoterápicos. Universidade Federal do Ceará, Departamento de Farmácia. https://farmacotecnica.ufc.br/wpcontent/uploads/2019/11/farmacot-fitoterapicos.pdf. 
Gosselin, R.E., H.C. Hodge, R.P. Smith, and M.N. Gleason. 1976. Clinical Toxicology of Commercial Products. Acute Poisoning, 4th ed. The Williams \& Wilkins Co., Baltimore, MD.

Hernández-Lambraño, R., Caballero-Gallardo, K., \& Olivero-Verbel, J. (2014). Toxicity and antifeedant activity of essential oils from three aromatic plants grown in Colombia against Euprosterna elaeasa and Acharia fusca (Lepidoptera: Limacodidae). Asian Pacific Journal of Tropical Biomedicine, 4(9), 695700. https://doi.org/10.12980/APJTB.4.2014APJTB-2014-0178

Hidayati, D., Darmanto, Y., Nurhidayati, T., \& Abdulgani, N. (2016). Short Communication: Larvicidal and antifeedant activities of Kalanchoe daigremontiana against Plutella xylostella larvae. Nusantara Bioscience, 8(2), 312-315. https://doi.org/10.13057/nusbiosci/n080229

Huang, S. H., Xian, J. D., Kong, S. Z., Li, Y. C., Xie, J. H., Lin, J., Chen, J. N., Wang, H. F., \& Su, Z. R. (2014). Insecticidal activity of pogostone against Spodoptera litura and Spodoptera exigua (Lepidoptera: Noctuidae). Pest Management Science, 70(3), 510-516. https://doi.org/10.1002/ps.3635

Josué, W. P., Payne, V. K., Alidou, M. N., Claire, K. M., Jeannette, Y., Gertrude, M. T., Mbida, M., \& Bilong, C. F. B. (2012). In vitro ovicidal and larvicidal activities of aqueous and ethanolic extracts of stem bark of Nauclea latifolia (Rubiaceae) on Heligmosomoides bakeri (Nematoda, Heligmosomatidae). Medicinal Plants, 4(4), 212-217. https://doi.org/10.5958/j.0975-4261.4.4.034

Karimzadeh, J., \& Rabiei, A. (2020). Larvicidal and oviposition deterrent effects of the jimsonweed (Datura stramonium L.) extracts on the diamondback moth, Plutella xylostella (L.). J. Agr. Sci. Tech., 22(5), 1279-1293.

Liu, S., Wang, X., Xu, Y., Zhang, R., Xiao, S., Wang, Y., \& Zhang, L. (2019). Antifeedant and ovicidal activities of ginsenosides against Asian corn borer, Ostrinia furnacalis (Guenee). PLoS ONE, 14(2), 1-14. https://doi.org/10.1371/journal.pone.0211905

Maroneze, D. M., \& Gallegos, D. M. H. N. (2009). Effect of Melia azedarach aqueous extract on the development of immature and reproductive stages of Spodoptera frugiperda (J. E. Smith, 1797) (Lepidoptera: Noctuidae). Semina:Ciencias Agrarias, 30(3), 537-550. https://doi.org/10.5433/16790359.2009v30n3p537

Matharu, K. S., \& Mehta, P. K. (2018). Antifeedant and ovipositional deterrent activity of medicinal plants of Western Himalaya on Plutella xylostella. Journal of Environmental Biology, 39, 966-972. https://doi.org/http://doi.org/10.22438/jeb/39/6/MRN-559

Matos, F. J., Lorenzi, H., Santos, L. F. L., Matos, M. E. O., Silva, M. G. V., \& Sousa, M. P. (2011). Plantas Tóxicas: estudo de fitotoxicologia química de plantas brasileiras. Nova Odessa: Instituto Plantarum.

Matsuura, H. N., Poth, A. G., Yendo, A. C. A., Fett-Neto, A. G., \& Craik, D. J. (2016). Isolation and Characterization of Cyclotides from Brazilian Psychotria: Significance in Plant Defense and Co-occurrence with Antioxidant Alkaloids. Journal of Natural Products, 79(12), 3006-3013. https://doi.org/10.1021/acs.jnatprod.6b00492

Miller, A. L. E., Tindall, K., \& Leonard, B. R. (2010). Bioassays for monitoring insecticide resistance. Journal of Visualized Experiments, 46, 3-7. https://doi.org/10.3791/2129

Miresmailli, S., \& Isman, M. B. (2014). Botanical insecticides inspired by plant-herbivore chemical interactions. Trends in Plant Science, 19(1), 29-35. https://doi.org/10.1016/j.tplants.2013.10.002

Mpumi, N., Machunda, R. L., Mtei, K. M., \& Ndakidemi, P. A. (2020). Insecticidal efficacy of Syzygium aromaticum, Tephrosia vogelii and Croton dichogamus extracts against Plutella xylostella and Trichoplusia ni on Brassica oleracea crop in Northern Tanzania. AIMS Agriculture and Food, 6(1), 185-202. https://doi.org/10.3934/agrfood.2021012

Ningsih, F. K., Aditiya, A. P., \& Prijono, D. (2020). Laboratory effectiveness of some insecticides and Piper aduncum fruit extract against diamondback moth (Plutella xylostella [L.]) from Cisarua-Bogor and Pangalengan-Bandung, West Java, Indonesia. IOP Conference Series: Earth and Environmental Science, 468(1). https://doi.org/10.1088/1755-1315/468/1/012003

Pena, M. R. (2012). Bioatividade de extratos aquosos e orgânicos de diferentes plantas inseticidas sobre a mosca-negra-dos-citros, Aleurocanthus woglumi Ashby 1915 (Hemiptera: Aleyrodidae). Tese de Doutorado, Universidade Federal do Amazonas, Manaus, Amazonas, Brasil.

Peres, L. L. S., Sobreiro, A. I., Couto, I. F. S., Silva, R. M., Pereira, F. F., Heredia-vieira, S. C., Cardoso, C. A. L., Mauad, M., Scalon, S. P. Q., Verza, S. S., \& Mussury, R. M. (2017). Chemical compounds and bioactivity of aqueous extracts of Alibertia spp. in the control of Plutella xylostella L. (Lepidoptera: Plutellidae). Insects, 8(125). https://doi.org/10.3390/insects8040125

Prakash, A., \& Rao, J. (1997). Botanical pesticides in agriculture. Boca Raton: CRC Press Inc.

Ribeiro, L. D. P., Ansante, T. F., \& Vendramim, J. D. (2016). Effect of ethanolic extract from Annona mucosa seeds on development and feeding behavior of Spodoptera frugiperda. Bragantia, 75(3), 322-330. https://doi.org/10.1590/1678-4499.473

Risco, G. V. S., Idrogo, C. R., Kato, M. J., Díaz, J. S., Armando-Jr, J., \& Paredes, G. E. D. (2012). Larvicidal activity of Piper tuberculatum on Spodoptera frugiperda (Lepidoptera: Noctuidae) under laboratory conditions. Revista Colombiana De Entomologia, 38(1), 35-41.

Sachs, I. (2009). Revolução tripla. Página 22, (34), 31-31.

Santiago, G. P., Pádua, L. E. de M., Silva, P. R. R., Carvalho, E. M. S., \& Maia, C. B. (2008). Effects of plant extracts on the biology of Spodoptera frugiperda (J. E. Smith, 1797) (Lepidoptera: Noctuidae) maintained under artificial diet. Ciencia e Agrotecnologia, 32(3), 792-796. https://doi.org/10.1590/s141370542008000300013

Santos, M. S., Zanardi, O. Z., Pauli, K. S., Forim, M. R., Yamamoto, P. T., \& Vendramim, J. D. (2015). Toxicity of an azadirachtin-based biopesticide on Diaphorina citri Kuwayama (Hemiptera: Liviidae) and its ectoparasitoid Tamarixia radiata (Waterston) (Hymenoptera: Eulophidae). Crop Protection, 74, 116123. https://doi.org/10.1016/j.cropro.2015.04.015 
Research, Society and Development, v. 10, n. 14, e19101421474, 2021

(CC BY 4.0) | ISSN 2525-3409 | DOI: http://dx.doi.org/10.33448/rsd-v10i14.21474

Shaidi, F., \& Naczk, M. (1995). Food phenolics: sources, chemistry, effects and applications. Lancaster: Technomic Pub. Co.

Silva, C. G. V, Oliveira, J. C. S., \& Camara, C. A. G. (2018). Insecticidal activity of the ethanolic extract from Croton species against Plutella xylostella L. (Lepidoptera: Plutellidae). Revista Facultad Nacional de Agronomía, 71(2), 8543-8551. https://doi.org/10.15446/rfna.v71n2.62881

Silva, D. R., Oliveira, T. A., Amaral, L. V., Fachin Espinar, M. T., Ronchi Teles, B., \& Nunez, C. V. (2013). Avaliação da atividade inseticida e antibacteriana do extrato metanólico dos galhos de Psychotria poeppigiana Mull Arg (Rubiaceae). Congresso Brasileiro de Quimica, 1(1), 1-1.

Silva, V. C., Giannini, M. J. S., Carbone, V., Piacente, S., Pizza, C., Bolzani, V. S., \& Lopes, M. N. (2008). New antifungal terpenoid glycosides from Alibertia edulis. Helvetica Chimica Acta, 91, 1355-1362.

Souza Júnior, A. A., Grazziotti, G. H., Petacci, F., Freitas, S. S., Tavares, W. S., Pereira, A. I. A., \& Cruz, I. (2011). Efeito inseticida de extratos etanólicos do caule de Psychotria goyazensis, Psychotria hoffmannseggiana e Psychotria prunifolia sobre lagartas e ovos de Spodoptera frugiperda. Horticultura Brasileira, $29(2), 465-472$

Sparks, T. C., \& Nauen, R. (2015). IRAC: Mode of action classification and insecticide resistance management. Pesticide Biochemistry and Physiology, 121, 122-128. https://doi.org/10.1016/j.pestbp.2014.11.014

Tavares, W. S., Grazziotti, G. H., Souza, A. A., Sousa Freitas, S., Consolaro, H. N., Ribeiro, P. E. A., \& Zanuncio, J. C. (2013). Screening of extracts of leaves and stems of Psychotria spp. (Rubiaceae) against Sitophilus zeamais (Coleoptera: Curculionidae) and Spodoptera frugiperda (Lepidoptera: Noctuidae) for maize protection. Journal of Food Protection, 76(11), 1892-1901. https://doi.org/10.4315/0362-028X.JFP-13-123

Trindade, R. C. P., de Souza Luna, J., de Lima, M. R. F., da Silva, P. P., \& Santana, A. E. G. (2011). Larvicidal activity and seasonal variation of Annona muricata (Annonaceae) extract on Plutella xylostella (Lepidoptera: Plutellidae). Revista Colombiana de Entomologia, 37(2), $223-227$.

Valli, M., Young, C. M., \& Bolzani, S. (2016). A beleza invisível da biodiversidade: o táxon Rubiaceae. Rev. Virtual Quim., 8(1), 296-310. https://doi.org/10.5935/1984-6835.20160020

Zalucki, M. P., Shabbir, A., Silva, R., Adamson, D., Liu, S. S., \& Furlong, M. J. (2012). Estimating the economic cost of one of the world's major insect pests, Plutella xylostella (Lepidoptera: Plutellidae): just how long is a piece of string? Journal of Economic Entomology, 105(4), 1115-1129. https://doi.org/10.1603/EC12107 